\section{EL DISCURSO DE LA INNOVACIÓN EN TELA DE JUICIO: TECNOLOGÍA, MERCADO Y BIENESTAR HUMANO}

\author{
José Luís García \\ Instituto de Ciências Sociais da Universidade de Lisboa
}

\begin{abstract}
The main trend in sociological studies of innovation has a positive outlook on innovation, bearing no distinction from the political and economic discourses that conceive it as an end in itself or as means at the service economic and commercial production. This article questions such vision and attempts to map out the main assumptions and factors that explain the dynamics of innovation as framed by the ongoing transformations of the current world. It suggests a focus on social studies in which innovation is interpreted as a social action, with its own ends, articulated with other activities of human life and, therefore, susceptible to ethical and moral valorization. Such focus would allow the clarification of the social and historical meaning of innovation processes as well as to extend them in the vast plan of unforeseen consequences, risks and uncertainties for society, human existence and the global eco-system.
\end{abstract}

KEY WORDS: Technoscientific innovation; commodification; knowledge economy; world self-regulated market system; techno-economic determinism; corporations; biocapital.

\section{INTRODUCCIÓN}

Este artículo realiza una reflexión crítica sobre el discurso de la innovación. En la adhesión irreflexiva de las orientaciones vigentes de la innovación está patente la creencia de que los avances tecnológicos son la concreción de un plan ejecutado en la historia del conocimiento, un plan benéfico que ilustraría una supuesta marcha ascendente de la racionalidad. Confrontando esta visión, el argumento que exponemos en este texto es que el actual régimen de innovación, más que estar orientado hacia la promoción del bienestar humano, está dirigido a colocar nuevos dominios bajo el control de la esfera productiva y de la economía de mercado. En la nueva fase de la economía y del capitalismo, dominios como la ciencia, las tecnologías de la información y las formas de vida biológica, entre varios otros, pasarán a estar crecientemente sometidos a
THE INNOVATION DISCOURSE ON TRIAL: TECHNOLOGY, MARKET AND WELLBEING

RESUMEN: La tendencia principal de los estudios sociológicos de la innovación la asumen como algo positivo, sin distinguirse de los discursos políticos y económicos dominantes que la conciben como un fin en sí mismo o un medio al servicio de la producción económica y mercantil. Este artículo cuestiona tal visión y busca trazar los presupuestos y factores más importantes que explican la dinámica de la innovación en el marco de las transformaciones en curso en el mundo actual. Se sugiere un enfoque para los estudios sociales en que la innovación sea interpretada como acción social, dotada de fines, articulada a otras actividades de la vida humana y, por lo tanto, susceptible de valoraciones éticas y morales. Un enfoque de este tipo permitiria esclarecer el sentido social e histórico de los procesos de innovación y también entenderlos en el plano amplio de sus consecuencias imprevistas, riesgos e incertidumbres para la sociedad, la existencia humana y el ecosistema global.

PALABRAS CLAVE: Innovación tecnocientífica; mercantilización; economía del conocimiento; sistema de mercado autorregulado mundial; determinismo tecnoeconómico; corporaciones; biocapital.

la lógica empresarial y al propósito de obtener y maximizar las ganancias. Para la institucionalización del régimen de innovación ha sido crucial la reestructuración de la actividad científica en tecnociencia guiada por estrategias mercantiles. Las novedades tecnológicas que resultan de la tecnociencia mercantil se han convertido en un componente central de la economía del mundo contemporáneo, pero esas innovaciones requieren inversiones cuantiosas en investigación y conocimiento, más allá de implicar nuevos problemas y peligros.

El presente artículo está organizado en cuatro puntos. Después de la introducción, el primer punto busca iluminar las reminiscencias de la noción moderna de progreso de los siglos XVIII y XIX, que constituyen la base para la admisión acrítica de la innovación. En la historia de la tecnología se puede vislumbrar un sentido que incorporaría el espíritu de 
la razón. Esta visión que, a pesar de sus equívocos, perseguia finalidades humanas para las invenciones, dio lugar a otra -expuesta en el segundo punto- que concibe la innovación como un régimen que precede a la mercantilización de vastos sectores del conocimiento, de la ciencia y de la tecnologia (en particular, a través del sistema de patentes) para, a su vez, abrir nuevos campos de mercantilización. Lo nuevo implica, por consiguiente, tanto una nueva estructura entre ciencia, tecnología y mercado, como una nueva economía. En el tercer punto se presentan los trazos de esta nueva economía. Una característica esencial del nuevo contexto económico reside en el surgimiento de industrias de base informacional (biotecnología, industria farmacéutica, semillas) o puramente informacionales (computadores, medios digitales) que operan a escala global y donde se articulan la sustentación del conocimiento científico-tecnológico, la apropiación de la función de reproducción y el control cerrado sobre la distribución y el uso para impedir la reproducción. En el cuarto punto mostramos cómo este orden económico, conjugado con la "financiarización" de las economías, tiende a sobreponerse a todos los otros órdenes, jurídicos, políticos, sociales y éticos. Es en este sentido que se torna posible afirmar que la estructura tecnoeconómica condiciona fuertemente todo lo demás en nuestras sociedades. El artículo concluye con una defensa de la idea de que los estudios sociales deben tener en consideración no sólo el análisis de las consecuencias sociales de la innovación, sino de que, sobretodo, deben aclarar el sentido de las estrategias tecnocientíficas y las relaciones que establecen con los valores.

\section{INNOVACIÓN Y LOS ECOS DE LA IDEA MODERNA DE PROGRESO}

Desde mediados de los años 80 del siglo $\mathrm{XX}$, un conjunto de líderes empresariales y políticos, acompañados de figuras y grupos provenientes sobre todo de los ámbitos de la gestión, de la economía y de la tecnología, comenzó a promover intensamente a escala mundial una noción anunciada como motor de las sociedades, la "innovación". En las declaraciones de estos dirigentes, el término innovación surge generalmente asociado a una idea entusiasta de las novedades técnicas e impulsora del dinamismo económico'. Los promotores de la innovación buscan implantar este concepto justificándolo con el papel que juegan los logros científico-técnicos en el cambio económico y en las consecuencias que puede tener en el bienestar humano². En sus discursos se encuentran constantes alusiones a la importancia de la innovación como agente de prosperidad económica y potenciador de numerosos beneficios para la vida humana y social. Tal discurso es ampliamente reproducido por las universidades, sobre todo en los cursos de gestión, a menudo de forma irreflexiva en cuanto a las funciones y consecuencias de las tecnologías.

Desde el punto de vista de los patrocinadores de la innovación resuenan las reminiscencias de las teorías del progreso de los siglos XVIII y XIX, basadas en una visión de la historia en la que ésta surgía como la realización de un proyecto racional, grandioso y benevolente. Hoy sabemos, a través de la reflexión filosófica, histórica y sociológica desarrollada a lo largo del siglo XX, que las ideas de los principales representantes del liberalismo, así como de pensadores con otras convicciones como Saint-Simon o Comte, aun manteniendo sus desacuerdos en otros temas, estaban impregnadas por una concepción providencialista de la historia. Ésta veía el cambio técnico como un medio deseable para alcanzar la meta de prosperidad que constituiría como la culminación de la evolución histórica. La misma mirada impregnaba el proyecto de Karl Marx, un crítico implacable de la forma que estaban tomando las sociedades occidentales del siglo XIX y un inspirador de la que se convirtió en la filosofía política más importante a la hora de confrontar a esas sociedades. En su visión, se constata la confianza en el supuesto de que el capitalismo sólo sería bien interpretado en tanto etapa de una lógica histórica en la que el desarrollo de las fuerzas productivas tenía como corolario esperable la edificación futura de una sociedad que procedería a una distribución equitativa de los bienes.

El pensamiento social está colocado en un dilema que surge de la frustración de los objetivos que movian a la concepción providencialista de la historia. Las nuevas expectativas están centradas en la tecnología. Todos los sueños que las tecnologías anteriores no realizaron se ven compensados por nuevas expectativas puestas en las tecnologías emergentes. Las tecnologías de un período dado son siempre presentadas como superiores a las del pasado. Cada producto de la innovación tecnológica sirve para anunciar un paso adelante en la lucha por una sociedad de la abundancia y por una vida humana guiada por la 
salud. Cada novedad tecnológica es promovida como un antecedente que prefigura un mundo mejor. Las lagunas y los fracasos de las tecnologías anteriores resultan inconvenientes al servicio de un final feliz. Las conquistas tecnológicas se nos presentan como una fuerza que torna activa la realización por parte de los seres humanos de sus expectativas. Las tecnologías son recibidas con una receptividad acritica $y$, aunque existan disidentes, su voz no ha sido en el pasado ni es en el presente suficiente para cambiar el curso de la construcción de una civilización tecnológica. Sin embargo, vale la pena pensar si es correcto ver la Historia de la tecnología en un horizonte evolutivo, acumulativo, lineal, como si obedeciera a una necesidad interna y a una sucesión de etapas considerada necesaria o inevitable. 0 incluso si cada tecnología obedece al cumplimiento de un itinerario o de un plano que anticipa lo esperado. Estos y otros presupuestos son eco de ideas que marcan las principales narrativas del último siglo y medio -una mentalidad prospectiva, optimista, voluntarista, que valora siempre el presente como superior al pasado, que celebra la novedad como paso para alcanzar el horizonte de perspectivas que ha construido.

Un análisis más atento sobre la perspectiva de los actuales impulsores de la innovación revela, sin embargo, un matiz digno de nota respecto de las teorias del progreso de los siglos XVIII y XIX. Más allá de la contribución al bienestar humano, los conceptos de innovación y de cambio económico son defendidos en la actualidad como valor absoluto e indiscutible. La idea que destaca en este punto de vista es que la innovación y el cambio tecnoeconómico deben celebrarse en sí mismos, ser adoptados sin vacilaciones y con rapidez, independientemente de las discusiones sobre cuáles son las implicaciones y beneficios prácticos de una determinada tecnología, las opciones disponibles en los modos de utilizar ciertos artefactos, o cuáles son los efectos de un determinado sistema para la vida colectiva.

Es verdad que, a mediados del siglo XIX, la alianza entre ciencia y tecnología ayudó a instaurar la convicción de que el bienestar humano estaba articulado estrechamente con el cambio tecnológico, expectativa que, en realidad, nunca fue verdaderamente cuestionada por los movimientos socialistas. Existía una confianza más o menos extendida de que los avances tecnológicos ayudarian a la humanidad a superar muchas de sus carencias y debilidades. Sin embargo, estos proyectos modernistas consideraban la tecnología como un medio al servicio del ser humano. Le correspondería a él guiarla de modo racional y subordinado a los valores de bienestar y felicidad de la humanidad.

\section{LA CONCEPCIÓN DE LA INNOVACIÓN EN EL ACTUAL CONTEXTO POLÍTICO Y ECONÓMICO}

En los actuales líderes mundiales del cambio tecnológico, es este tipo de pensamiento y modo de concebir la tecnología el que parece haber terminado. Del modo antiguo sólo ha quedado el débil eco del providencialismo histórico, ahora transformado en un plan en el que la innovación tecnocientífica debe ser guía y destino de la historia. Desde el punto de vista de los decisores de nuestro tiempo, resulta superflua cualquier discusión sobre la relación entre la tecnología y la estructura moral de las sociedades contemporáneas, o sobre los riesgos, incertidumbres, subproductos y desenlaces imprevistos del cambio tecnológico. No se sienten tampoco atraídos por la discusión sobre formas alternativas de organización social y el peso diferente que en ellas podrian tener otros sistemas tecnológicos más adecuados para propiciar el bienestar humano. Los límites al desarrollo tecnológico, muchas veces entendido como dispositivo negativo más que como un principio constructivo, se agotan en el discurso ambientalista ya institucionalizado.

El liberalismo económico defendido por esa élite mundial ha mostrado una firme tendencia a abrazar, de forma casi ilimitada, el culto a la innovación, impulsado por las oportunidades de los ciclos de negocios posteriores a las innovaciones y por la defensa de que sólo la lógica del mercado debe decidir qué opciones deben prevalecer ${ }^{3}$. Despojadas de la creencia decimonónica de que existía un movimiento para la prosperidad universal, las antiguas teorías del progreso fueron sustituidas, en diversos períodos del siglo XX, por términos tales como "riqueza económica", "crecimiento económico" e "innovación". La producción opulenta y omniampliada de mercancías, así como la incitación al consumo mediante la movilización del deseo y del gusto, se convirtió en la cara desfigurada de los ideales de bienestar y felicidad.

Marx fue el primero que percibió con profundidad que el liberalismo impulsaba el fenómeno de la mercantilización

ARBOR Vol. 188753 enero-febrero [2012] 19-30 ISSN: 0210-1963 
(commodification, en inglés), la transformación de una cosa o relación en mercancía. La atención de Marx se centró principalmente en la transformación del trabajo en mercancía. Correspondió al historiador económico Karl Polanyi, en pleno siglo XX, analizar la transformación generadora del sistema de economía de mercado responsable de traer al interior de éste realidades ni siquiera producidas, como la tierra, o, que cuando lo eran, no se destinaban a la venta, como el caso del trabajo humano, denominando a esas mercancías "ficticias". Bajo la lógica del control del sistema económico por el mercado, como argumenta Karl Polanyi en su muy celebrado libro La Gran Transformación, publicado en 1944, cantidades crecientes de ámbitos, gran parte de ellos desbravados por la tecnología moderna (por la "edad de la máquina", según sus palabras), se transformaban en mercancías, constituyéndose de este modo una esfera económica que se habría desmarcado de otras instituciones en la sociedad y que se convirtió en determinante para la vida social.

Según Polanyi, en la medida en que ningún conjunto humano puede sobrevivir sin un sistema de producción, su anexión en un dominio institucional delimitado y diferente de la sociedad tuvo como consecuencia convertir al resto de la sociedad cada vez más heterónima frente a esa estructura. La configuración social que ha resultado de este gran cambio histórico ha sido una sociedad que ha pasado a ser dirigida como si fuese un apéndice del mercado, una sociedad modelada de manera que el sistema funcione de acuerdo con las leyes del mercado. Como señala Polanyi en un pasaje muy citado, "en lugar de que la economía se sumerja en las relaciones sociales, éstas son las que se arraigan en el sistema económico" (2000: 77). El control del sistema económico-productivo por parte de la economía de mercado permitió que ésta pasase a ejercer dominio sobre los recursos de la naturaleza y sobre los seres humanos en sus actividades diarias. Polanyi ofrece la siguiente ilustración de ese control del mecanismo de mercado, y no se le escapa la interrelación con la tecnología moderna y el desarrollo de una economía orientada hacia el aumento de la producción, la demanda y del consumo: "Mientras que todos aquellos que carezcan de propiedad sean obligados a vender su trabajo para satisfacer su necesidad de alimento, y mientras que todos aquellos que tengan propiedades sean libres de comprar en los mercados más baratos y vender en los más caros, la ciega máquina seguirá arrojando cantidades siempre mayores de mercancías en beneficio de la raza humana. El temor de los trabajadores a la miseria y la avidez de los empleadores por lograr beneficios mantendrán en pie ese enorme aparato" (Polanyi, 1994).

Aunque un grupo de investigadores, conocido como "nueva sociología económica", haya presentado el argumento atractivo de que la historia de la formación de los mercados modernos no puede ser vista como totalmente desligada de la vida social y de los cambios y vínculos comunitarios ${ }^{4}$, tal y como aparecería en la perspectiva de Polanyi, son muy patentes en el mundo contemporáneo las devastadoras consecuencias de la construcción de un mercado global que se rige casi exclusivamente por la idea de lucro, desvinculado de cualquier idea o regulación de orden cívico.

\section{LO NUEVO Y LA NUEVA ECONOMÍA}

Desde las dos últimas décadas del siglo XX, la prosecución tenaz de la innovación se ha traducido en la asignación al sistema productivo y a la esfera de mercado de nuevos dominios que formaban parte de la organización biológica de los seres vivos, de la estructura de la materia y de los sistemas de conocimiento. Se observó en este periodo la tendencia a que las innovaciones se "agrupen", para usar un término de Schumpeter, abarcando las áreas de las tecnologías de la información (software, internet, móviles, nuevos medios), biotecnociencias (ingeniería genética o genómica, biología sintética, diversas áreas de la biotecnología y de las llamadas ciencias de la vida y de la salud) y nanotecnologías, entre otros campos. La convicción de que nos empuja un cambio científico y tecnológico articulado con características estructurales de la esfera económica se basa principalmente en el protagonismo adquirido por parte del conjunto de las nuevas industrias mencionadas. Es un hecho indiscutible que los nuevos dominios tecnológicos han estimulado cambios $y$, en muchos casos, han sustituido áreas significativas del contexto tecnológico anterior, mientras que proceden a la integración de varias otras ${ }^{5}$. Cabe mencionar también que el desarrollo de campos tecnológicos e industriales, como el de las tecnologías de la información y de las biotecnociencias, impulsa un nuevo ciclo de negocios y va acompañado por un entorno de perturbación, tanto en términos de orientación económica y política como ideológica. 
La importancia de la emergencia del nuevo contexto bajo la directriz de la idea de innovación está directamente relacionada con el tema de la transformación del capitalismo de "economía del trabajo" en "economía del conocimiento", como mecanismo clave de la acumulación del capital en un orden económico que ha tratado de tomar la forma de un sistema de mercado auto-regulado a escala mundial. En este aspecto, resulta importante hacer hincapié en que el conocimiento al que nos referimos no debe entenderse como restringido sólo al conocimiento científico. Las nuevas industrias emergen en los campos cognitivos y científico-tecnológicos en los que las nociones de información, comunicación y modelo de información/cibernético (que incluye pensar la vida biológica como organización informacional) por lo general tienen una importancia considerable. La explotación de los usos tecnológicos de la noción de información constituyó el motor del grupo de industrias cuyo ascenso se basa en los prometedores resultados obtenidos en áreas que incluyen tanto las tecnologías de la información, como las biotecnociencias basadas en la recombinación del ADN. El recurso a la potencialidad de los conceptos de información y a la integración de la información en el universo de las máquinas por parte de la cibernética permitió, por ejemplo, inaugurar una forma completamente nueva de pensar el fenómeno biológico, desarrollada en el período inicial de constitución de la biología molecular y para la que fue determinante el ambiente en torno a la mecánica cuántica, en particular a la tendencia que se interesó en el estudio de las cuestiones biológicas para aclarar las leyes de la física ${ }^{6}$. Si la teoría de la información proporcionó a la biología abundantes potenciales operativos, su adopción también ha generado nuevas e importantes dificultades, tales como las relativas a la difusión de la metáfora "programa genético" y a la perspectiva que veía en esta noción la fuente del desarrollo biológico ${ }^{7}$.

Otro elemento de cambio a finales del siglo XX fue el mercado financiero, que entró en una turbulenta dinámica de innovación cuyo vigor duró hasta el estallido de la crisis provocada por ese sistema en septiembre de $2008^{8}$. Procurando ser cada vez más competitivo, trató de atraer todos los ahorros y multiplicó los productos bancarios, al mismo tiempo que se sometió a reestructuraciones de los sistemas de alianzas y a la redefinición de la forma del servicio. El movimiento de "financiarización" de la economía se combinó con el declive de las formas de Estado- proveedor europeas, teniendo como telón de fondo el fin de la influencia del gasto público sobre la prosperidad económica y la ruptura del equilibrio intergeneracional, con la caída de la fecundidad y la acentuación del envejecimiento. El Estado fue perdiendo su papel capital en el desarrollo económico y en el bienestar social, lo que dejó al descubierto las dificultades del keynesianismo en el nuevo escenario. Se asentó la tendencia hacia la liberalización de las economías nacionales, influida por un cuerpo de doctrinas neoliberales que intentó aplicar el mercado como una fuerza modeladora de la sociedad en su conjunto, pasando su forma específica de organización a ser tendencialmente el patrón para la constitución de múltiples aspectos de la existencia humana.

La economía también ha ganado una configuración asociada a grandes entidades de poder privado a escala multinacional y transnacional. Las corporations internacionales se convirtieron en una de las fuerzas motrices de los procesos económicos, concibiendo y poniendo en acción estrategias con efectos en diferentes escenarios nacionales. En este nuevo contexto, emergió paralelamente un conjunto de esferas sociales transnacionales y una camada de agentes que abarcan la totalidad del sistema, superando muchas de las relaciones en la escala de los Estados-nación e incluso entre las naciones. Las autoridades reguladoras comenzaron a colaborar más estrechamente con las corporations, inclinándose a reducir las posibilidades de acción reguladora de los gobiernos nacionales y permitiendo la autorregulación de los grandes conglomerados empresariales.

Una constatación evidente es el papel extremadamente relevante jugado por las tecnologías de la información en el marco de este episodio espectacular. Lo que, en términos técnicos, posibilitó a los agentes transnacionales y nacionales desplazarse en el mercado mundial fue la existencia de una base instrumental en constante evolución, la cual garantiza el aumento de la capacidad de coger, reproducir, manipular y transmitir información de diversos tipos, así como modificar profundamente los modos de producción y distribución. Las nuevas tecnologías de la información también se encuentran en la génesis de profundos cambios en la economía internacional a través de un conjunto de innovaciones que impactaron en diversos aspectos de las economías y las sociedades del siglo XXI. Éste es el caso del movimiento de convergencia global entre las tecnologías de la información y las biotecnociencias. Las infraestruc- 
turas tecnológicas características de este movimiento sostienen que se recurra a las capacidades de procesamiento de los ordenadores y al uso de Internet para permitir el intercambio de datos biológicos a escala mundial. El ciclo de transformación del ADN, desde material biológico en un tubo de ensayo hasta la secuenciación del genoma como "bioinformación" computarizada y patentada con valor económico, presupone la interconexión entre ámbitos de la biología y de la informática, además de su operatividad a escala global. Este "capital-conocimiento biológico" o "biocapital", como entidad globalizada, es expresión de un sistema económico de mercado tendencialmente único y técnicamente interconectado, aunque heterogéneo y desigual ${ }^{9}$.

El surgimiento y desarrollo del sector industrial de las biotecnociencias ilustra un último e importante aspecto del contexto que estamos describiendo en términos de crecimiento económico: su dependencia del conocimiento científico. Hace más de dos siglos que el crecimiento sistemático de la riqueza ha dirigido el rumbo de las sociedades de occidente, un crecimiento que puede considerarse "científicamente sustentado", para utilizar una idea certera de Gellner (1995 [1992]), basada en el impulso constante que la ciencia permite dar a la innovación tecnológica y a la investigación industrial, es decir, a la creación y desarrollo de una civilización tecnológica. Esta comprensión del sentido general de las sociedades modernas esclarece la diferencia entre formaciones sociales con una prosperidad económica dentro de los límites de unas tecnologías simples y otras de crecimiento no limitado, basado éste en la automatización de la innovación científico-tecnológica y en su aplicación a esferas cada vez más amplias de la existencia humana (y que no se limitan sólo a ésta). Pero la ciencia, con cuyo apoyo floreció la tecnología de base científica, también se convirtió en el factor histórico que permitió tornar la innovación en gran medida desvinculada de las relaciones sociales y abrir el "camino real para el crecimiento económico perpetuo". Esta imagen contrasta con una modernización apoyada en la división del trabajo y en la tecnología precientífica, que sólo podría llevar a la humanidad hasta un cierto punto (Gellner, 1995).

Si la demanda de crecimiento económico ininterrumpido se caracteriza por la intensidad cada vez mayor de conocimiento científico y tecnológico (y también de otros factores como el marketing, el conocimiento sobre los mercados y los consumidores, y los recursos imaginativos y comunicacionales de la fuerza laboral) aplicado a los procesos de producción, los cambios no se producen sólo en el sector de la producción. Más allá de las implicaciones en la organización del mundo industrial y empresarial, el dinamismo de este proceso también ha provocado cambios sensibles en el propio campo científico, con respecto al cual no será inoportuno hablar de una posibilidad real de transfiguración. Esta metamorfosis ha tenido lugar con cierta nitidez a partir de la Segunda Guerra Mundial, pero sólo se viene afirmando sólidamente desde finales del siglo $X X$, implicando el propio status de la ciencia y dirigiéndola hacia una pérdida sustancial de su autonomía relativa frente al mundo industrial, comercial y al poder político.

La ciencia moderna se ha organizado como un espacio independiente con respecto a otros ámbitos, como la religión, la política y la economía, desde los siglos XVII y XVIII en Inglaterra, creando papeles científicos delimitados por principios internos reguladores de la actividad científica. El desarrollo y expansión de la ciencia no implicaron sólo la creación de teorías, modos operativos y la realización de descubrimientos, sino un proceso social de institucionalización en un contexto que le fue favorable. El análisis histórico de las relaciones entre ciencia y sociedad da a conocer un campo científico heterogéneo, que no es inmune a la influencia del poder, del comercio y del prestigio, y que se relaciona con los sectores sociales fuera de la esfera científica. La imbricación entre la ciencia, la tecnología y la industria es un elemento crucial de la constitución de

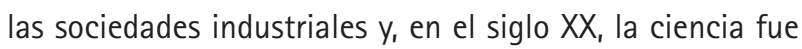
adquiriendo también un carácter cada vez más industrializado, que se hizo evidente en el período posterior a las Guerras Mundiales. Las grandes empresas pasaron a disponer de laboratorios en su interior y muchos laboratorios se han convertido también en empresas o pasaron a trabajar como empresas. La industria pasó a ser una entidad con un componente científico cada vez más intenso y sectores de la ciencia fueron adoptando una forma industrial. Las tesis que defienden la no linealidad de la innovación tecnológica, es decir, la interacción entre la investigación científica y otras áreas en la cadena de la innovación como el desarrollo de productos o el marketing, confirman plenamente la integración de la ciencia en los objetivos mercantiles ${ }^{10}$. La industria y diversos campos particulares de la ciencia y la tecnología establecieron sólidos lazos de carácter trans- 
nacional, a menudo con el apoyo e incentivo expreso de los Estados nacionales en los que están integrados.

La inscripción de las esferas científicas en la economía y en la competición de los mercados se reforzó como tendencia ideológica y como realidad en el marco del vigor neoliberal emergente en el curso histórico resultante de las crisis energéticas de los años 70 y de las depresiones económicas de finales de los años 80 . Aunque no existan suficientes estudios documentados que apunten el sentido de los cambios de reconfiguración del conjunto del campo científico y de su incorporación a la esfera industrial y comercial ${ }^{11}$, en áreas como las ciencias biológicas y médicas existen indicios considerables de la endogeneización de éstas en la esfera empresarial y en la lógica de la comercialización, así como de importantes cambios en el status y el mandato de los investigadores, muchos de los cuales viven bajo la presión de su conversión en productores de mercancías $^{12}$. La ciencia, de esfera relativamente autónoma inherente a las sociedades liberales y apoyada por las universidades y laboratorios públicos, ha estado sometida a una orientación que la integra en el sistema económico de mercado y en un campo subordinado a la capitalización del conocimiento, es decir, su transformación en el "capital conocimiento". Muchos agentes del mundo científico, unos entusiastas de la nueva misión que la ciencia y la enseñanza superior deben jugar en el crecimiento económico y otros indiferentes a lo que está en juego en las políticas de ciencia y tecnología, están abandonando la dimensión moral de su actividad, la que se traducia en la idea del conocimiento como bien público e independiente. Desde ese punto de vista, tiene sentido cuestionar los problemas asociados al aumento de la influencia de las grandes corporations en las decisiones de un sector sensible de la propia civilización liberal, el de las interrelaciones entre las ciencias, el Estado, el mercado y el espacio público. La presión ejercida por los gobiernos y por las corporations está llevando a la transformación en mercancias de sectores cada vez amplios de la universidad y de la investigación científica, y además está generando conflictos causados por los propios avances científicos y tecnológicos en áreas como los de nuestra relación con el fenómeno de la vida y con la naturaleza.

Con la fuerza que el liberalismo económico fue infundiendo, la lógica que pasó a predominar en las sociedades industriales de mercado tomó la configuración de un sistema de mercado auto-regulado en proceso incesante de expansión y profundización, cuyo vehículo reside en la capacidad que tiene el universo tecnológico de inscribir a los individuos en sus mecanismos de producción, consumo y cultura. Las posiciones que vislumbran la democratización de la innovación a través de la participación del consumidor en las nuevas tecnologías, señalando este desarrollo tecnológico como una emanación del ciudadano, de las que von Hippel (2006) es un ejemplo, tienden a pasar por alto que el discurso de la co-creación es una forma de dominio que utiliza realmente el trabajo del consumidor para fines empresariales (Zvick, 2008: 163). El fomento de la innovación tecnológica tiene como contrapartida la búsqueda de un alto grado de adopción social de los productos y resultados tecnológicos en general, regulado sólo por el mercado (o, con respecto a las armas, por la eficacia bélica autorizada por los Estados) y que prescinde de consideraciones de índole ética, filosófica o religiosa. El engranaje de la innovación tecnológica ha ido desarrollando el determinismo tecnológico como principio que rige las sociedades industriales, de la misma manera que el mecanismo de mercado, como señaló Polanyi ${ }^{13}$, tornó ampliamente al determinismo económico en un conductor de estas sociedades. Si bien es cierto que tanto el determinismo tecnológico, como el determinismo económico, no pasan de falacias cuando se consideran como una teoría de las sociedades humanas, no nos parece menos riguroso afirmar que, en el marco de las sociedades actuales, son estos determinismos los que están marcando muchas de las direcciones de nuestro rumbo social y el tipo de problemas y peligros a los que tenemos que enfrentarnos.

\section{HACIA UNA VISIÓN ALTERNATIVA DE LA INNOVACIÓN}

Los líderes mundiales que dirigen la economía y la política condujeron las últimas décadas del siglo XX de acuerdo con la orientación que concibe la tecnología y el mercado como el núcleo de la vida económica y social, negando la existencia de otro destino que no sea el subordinado a esta supremacía. Resuenan en esta orientación, como se ha señalado al principio de este artículo, ecos tenues $-y$ deformados- de la convicción defendida por diversas variantes del modernismo por la que la ciencia y la industria garantizarian el bienestar humano, y donde el progreso se articularía necesariamente con el avance tecnológico y el

ARBOR Vol. 188753 enero-febrero [2012] 19-30 ISSN: 0210-1963

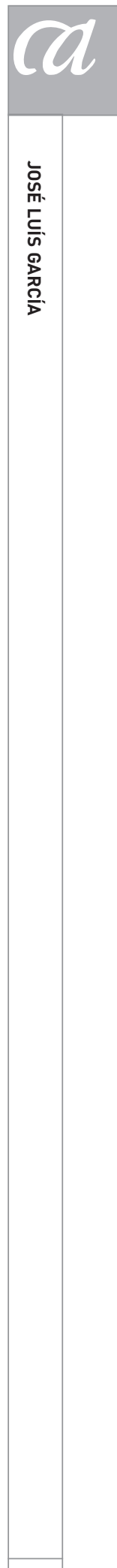


cambio económico. Sin embargo, a lo largo del siglo XX se verificó que gran parte de la innovación no sólo está conectada con los beneficios, sino con la destrucción bélica, ecológica y con la desigualdad social, que los avances tecnológicos integran poder económico y político, y que no implican sólo la invención, sino que también conducen al conflicto. Como escribe Salvador Giner, "los daños que él [capitalismo] causa -la pobreza que genera entre sus víctimas, la agonía progresiva del medio ambiente del que vivimos, la devastación bélica que directa o indirectamente fomenta- son resultado de invenciones cuyos creadores saben desde el primer instante para qué sirven, el daño que son capaces de causar" (2010: 128).

La orientación ideológica que ha condicionado el sentido de las sociedades desde los albores del siglo XXI trata de imponer el cambio tecnológico y las relaciones de mercado, no tanto por asociarlas a la posibilidad de tratarse de un factor para el bienestar humano general o para el progreso, sino por razones menos nobles y despojadas de ideales cívicos. Dejando de lado cualquier evaluación de las formas predominantes de la participación de la ciencia y de la tecnología en la economía de mercado del siglo XX, en los desastres ambientales y en las guerras, pasando por alto el carácter polémico de muchas osadías técnicas y sin querer tener conocimiento de los problemas que pueden implicar la endogenización de la universidad y de la ciencia a la economía liberal, la mentalidad de la innovación ha afirmado su poder frente a cualquier regulación o ponderación de su rumbo en términos sociales, ecológicos y políticos (descuidándose hasta el reconocido carácter estocástico que la innovación en sí comporta, lo que exige, por tanto, una mayor supervisión) ${ }^{14}$. Las élites dirigentes están convencidas de que somos seres económicos y mercantiles por naturaleza, negando así nuestro carácter primordial como seres sociales y simbólicos; no consiguen imaginar otro camino que no sea el de aumentar la capacidad de las máquinas y proseguir con el proyecto de dominar de forma ilimitada la naturaleza y buscar la riqueza económica infinita, y para eso tratan de evitar que los ciudadanos reflexionen sobre las decisiones de orientación de la universidad, sobre la política de investigación o sobre las directrices económicas.

La disposición general del período histórico que adquirió unos contornos cada vez más nítidos a partir de la Segunda Guerra Mundial adoptó un rostro cada vez más tecnoeconómico, y se encuentra en marcha una tendencia firme de fusión ciencia-tecnología-mercado que permanece ciega ante cualquier pregunta acerca de qué tipo de mundo está estimulando. En gran medida, la vida humana y social transcurre ahora en un contexto de sobredimensionamiento de la esfera de la técnica y el mercado para la mayor parte de la humanidad dependiente de los servicios técnicos (suministro de agua, luz, medios de transporte, comunicación, alimentación, etc.) que se sitúan a gran distancia de las comunidades, dominados por experts y empresas, y cuyos errores pueden significar situaciones de degradación, incertidumbre y ansiedad. También las acciones humanas, en particular las que tienen consecuencias sociales más penetrantes, se desarrollan principalmente a través de sistemas y conexiones técnicas sometidos a la lógica de la mercantilización.

Tomar conciencia de las consecuencias de la importancia de la esfera tecnoeconómica para la aceleración del proceso en el que las sociedades contemporáneas se ven envueltas no tiene que significar su aceptación. Por el contrario, puede ser una manera de insistir en la defensa de una idea de hombre y de sociedad que concede primacia a la esfera de las relaciones sociales, en contraposición a una perspectiva basada en la primacía de la tecnología y del sistema económico. Admitir en términos descriptivos e interpretativos que en las sociedades contemporáneas el cambio tecno-económico funciona como un motor que altera la estructura social, política, legal, el mundo de las artes, las creencias, las costumbres y escalas de valores, no implica la defensa, epistemológica o política, del determinismo económico y tecnológico. Aquellos que niegan que es la esfera tecnomercadológica la que ha estado condicionando en gran medida todo lo demás, aunque movidos por la defensa abstractamente intocable de la identidad social y política de los seres humanos, no contribuyen a que se perciba con claridad que sólo actuando políticamente es posible crear un mundo menos injusto socialmente, menos desequilibrado en sus relaciones con la naturaleza y más reflexivo sobre el poder de los dispositivos técnicos.

La corriente del "constructivismo social de la tecnología" tiene como proyecto estudiar los distintos modos en los que los sistemas y artefactos tecnológicos se entrelazan con el contexto social. Varias voces de la "nueva sociología económica" también tratan de mostrar cómo los mercados se incluyen en la vida social. Aunque estas iniciativas 
constituyen perspectivas y agendas de investigación completamente legítimas para la generación de conocimiento sociológico, no dispensan, sin embargo, de la existencia de otras perspectivas y proyectos más centrados en el cuestionamiento de las opciones en materia de investigación, de las decisiones sobre los sistemas tecnológicos, así como de los ámbitos en los que el mercado se debe subordinar a la regulación pública ${ }^{15}$. En nuestras "sociedades de mercado", más que una teoría equivocada del mundo social, el determinismo tecnoeconómico es un hecho. La constatación de que el determinismo tecnoeconómico está condicionando poderosamente todo el resto es la única forma de tener claro que sólo la conciencia ética y la acción política pueden ser los medios para alterar la esfera tecnoeconómica y su relevancia en la vida social.

\section{Conclusión}

Las sociedades tienen ante sí la responsabilidad de hacer frente a problemas de enorme magnitud generados por las tendencias descritas, destacando entre ellos los inherentes a esta civilización tecnológica, como la crisis ecológica global, el espectro de las guerras nucleares y la posibilidad del control biológico del ser humano -y también la mercantilización de las formas de vida, de las más simples a las más complejas- a través de la ingeniería genética. Paralelamente, el mundo contemporáneo mantiene, al menos para la gran mayoría de la humanidad, muchos de los graves problemas de escasez y enfermedad que tenía al principio de las sociedades industriales. La industria, la ciencia y la tecnología, cuyos éxitos jugaron innegablemente un papel en la mejora de las condiciones de vida de los seres humanos, se han convertido en una fuente de dificultades e incertidumbres en un sistema que está actualmente bajo el impulso del ultra-liberalismo económico y del proyecto de construcción de un mercado mundial auto-regulado. El régimen de innovación permanente como motor de crecimiento económico, de la construcción de mercados de futuros biológicos $y$, en otros dominios, de la constitución de un biocapital, o incluso como medio de descubrimiento al servicio del poder, de la violencia y de la guerra, expone a la humanidad no sólo a cuestiones morales y políticas totalmente desconocidas, sino sobre todo a un horizonte inédito de amenazas y peligros obra de los propios seres humanos. Usando las palabras de Lamo de Espinosa, la innovación tecnológica es hoy un régimen de "invenciones sin fin, es decir, sin finalidad, sin objeto" (2010: 69). Además, todo esto se produce en una circunstancia en la que el elemento político surge con una imagen de debilidad e irrelevancia en lo que respecta al debate y a la toma de posiciones adecuadas sobre los problemas planteados por las nuevas formas de poder.

Moldear la sociedad al sistema tecno-económico vigente, para conservarlo sin cambios importantes, ha sido la intención de las oligarquías que dirigen el mundo. Intervenir de forma consciente y responsable en este sistema, para abrirlo a la discusión pública y ponerlo al servicio del bien común, ha sido defendido por aquellos que creen en una sociedad verdaderamente democrática. Pero incluso esta segunda opción, para que tenga éxito, debe estar ahormada por una concepción del ser humano y de la sociedad muy diferente a la legada por la creencia en la tecnología y la economía de mercado como fines últimos de la vida humana.

\section{NOTAS}

1 A tales concepciones no serán ajenas las tesis de la primera mitad del siglo XX del economista Joseph Schumpeter, según las cuales la innovación tecnológica es endógena y esencial para el desarrollo económico, y no un factor externo (Schumpeter, 1996: 125).

Recibido: 4 de enero de 2011

Aceptado: 10 de junio de 2011
2 Siendo posible distinguir innovación de productos, innovación de procesos e in- cluso innovación en términos de organización, en este texto nos asomamos a su dimensión tecnocientífica, es decir, la referida a los procesos y productos que integran a la ciencia y la tecnología con ámbitos económicos y sociales.

3 Por ejemplo, la adopción de un Sistema Nacional de Innovación, un concepto desarrollado por Freeman (1995: 5-24), entre otros, parece ser dominada por las exigencias del 
mercado, donde el Estado participa con pocas preocupaciones más allá de ésa, y donde la tecnología emerge como un valor en sí misma.

4 En este sentido, véase específicamente Bruni y Zamagni (2007). La "nueva sociología económica" incorpora una amplia diversidad de enfoques que buscan estudiar el meollo de las problemáticas económicas, transponiendo los supuestos que han guiado la economía convencional. El Handbook of Economic Sociology, organizado por Smelser y Swedeberg, editado originalmente en 1994, y otras obras de autores como Granovetter (1990), aparecen como ejemplos de este emprendimiento con una posición teórica poco clara. Para un cuestionamiento del status de la "nueva sociología económica", véase Ferreira et al. (1996) y Graça (2005: 111-129).

5 A este respecto, parece oportuno recordar el concepto de "paradigma tecnológico" que desarrolla el economista Dosi (1992: 147-162), un concepto que designa una serie de prácticas tecnológicas determinadas por un modelo (el microprocesador, por ejemplo) que define el tipo de problemas que hay que resolver y la trayectoria tecnológica que hay que seguir. En este sentido, se puede evocar también la noción de "destrucción creativa", popularizada por Schumpeter (1976), en la medida en la que designa el modo cómo lo nuevo sustituye a lo antiguo.

6 El modelo de la información aplicado a la biología basó sus hipótesis en las obras de Schrödinger, What is Life (1989 [1944]), de Shannon y Weaver, The Mathematical Theory of Communication (1948) y de Norbert Wiener, Cybernetics (1948), que contribuyeron considerablemente a la teorización y aplicación del concep- to cibernético de "sistema" regulado por la transmisión de información.

7 A este respecto, tiene sentido recordar que, no sin resistencia manifiesta de muchos bioquímicos y biólogos dedicados a la investigación estructural, acompañados de otros científicos, el concepto cibernético se convirtió, en primer lugar, en la noción básica de todo aquel nuevo campo científico y que, después, tuvo un impacto en las más diversas áreas, y no sólo en las ingenierías y en las ciencias físico-naturales, en particular a partir del desarrollo de las tecnologías de la información y computación. Con respecto a una literatura reflexiva en este campo, véase, entre otras obras importantes, Jorge (1995), Lewontin (1998), Oyama (2000), Keller (2000) y Leite (2006).

8 El estallido de la llamada burbuja "punto com", en abril de 2000, fue una crisis especialmente relacionada con la creencia en la innovación como generadora de crecimiento económico sin fin y sin límites: la creencia en un nuevo paradigma tecnológico como productor de riqueza.

9 Para una discusión sobre el concepto de "biocapital", véase Thacker (2005), Garcia (2006a: 981-1009) y Sunder Rajan (2006).

10 Véase Kline y Rosenberg (1986: 275305).

11 Entre la ya relativamente considerable literatura que discute este tema véase Gibbons et al. (1994), Ziman (1994; 1996), Weingart (1997), Krimsky (2003), Pestre (2003), Nowotny et al. (2001; 2005), Shinn y Ragouet (2008), Garcia y Martins (2008: 397-417).

12 A este respecto, véase, entre otros, García (2006a; 2006b) y GuespinMichel y Jacq (2006).

13 La posición de este autor sobre este tema está bien expuesta en Polanyi (1994).
14 Cabe señalar que Schumpeter diferenció invención de innovación, siendo la primera la creación del producto y la segunda el producto que tiene éxito, aquél que se adapta a la sociedad. Este último, elemento central en este artículo, es por sí solo problemático, porque en la actual "sociedad de mercado" éxito significa consumo; lo cual no es necesariamente el mejor criterio. Lo ideal sería, entrando en el registro político, que el bien común fuese el criterio de "éxito". Aunque carente de demostración, la práctica corriente de los programas de incentivos para la innovación parece guiarse, por ahora, por la omisión relativa al bien común.

15 Una valiosa contribución a una reflexión sobre la noción de determinismo tecnológico es la de Bimber (1990: 333-351), en particular, la distinción que hace entre el determinismo tecnológico "nomológico", "normativo" y de las "consecuencias no deseadas". En este artículo, que trata la interpretación del sistema, nos referimos al normativo (la creencia en el determinismo como una promesa y necesidad) y al de las consecuencias no deseadas (incluido en el discurso de la incertidumbre), rechazándose lo nomológico, "leyes que rigen las sociedades humanas".

\section{BIBLIOGRAFÍA}

Bimber, Bruce (1990), "Karl Marx and the three faces of technological determinism", Social Studies of Science, 20: 333-351.

Bruni, Luigino y Zamagni, Stefano (2007), Civil Economy Efficiency, Equity, Public Hapiness, Oxford, Peter Lang.

Dosi, G. (1992), "Technological paradigms and technological trajectories. A suggested 
interpretation of the determinants and directions of technical change", Research Policy, 11 (3): 147-162.

Fagerberg, Jan et al. (2005), The Oxford Handbook of Innovation, Oxford, Oxford University Press.

Ferreira, José M. Carvalho; Marques, Rafael; Peixoto, João y Raposo, Rita (orgs.) (1996), Entre a Economia e a Sociologia, Oeiras, Celta.

Freeman, C. (1995), "The national system of innovation in historical perspective", Cambridge Journal of Economics, 19: 5-24.

Garcia, José Luis (2006a), "Biotecnologia e biocapitalismo global", Análise Social, vol. XLI, 181: 981-1009.

Garcia, José Luís (2006b), "As tecnociências da vida e as ameaças do eugenismo e da pós-humanidade", en A. P. Pita, J. L. Garcia, L. A. Costa Dias e P. Granjo, Quatro Olhares sobre a Cultura, Barreiro, Cooperativa Cultural Popular Barreirense.

Garcia, José Luís y Martins, Hermínio (2008), "O ethos da ciência e as suas transformações contemporâneas, com especial atenção sobre a biotecnologia", en Manuel Villaverde Cabral et al., Itinerários. A Investigação nos 25 Anos do ICS, Lisboa, Imprensa de Ciências Sociais, 397-417.

Gellner, Ernest (1995 [1992]), Razão e Cultura: papel histórico da racionalidade e do racionalismo, Lisboa, Teorema.

Gibbons, Michael et al. (1994), The New Production of Knowledge: the dynamics of science and research in contemporary societies, London, Sage.

Graça, João Carlos (2005), "Afinal, o que é mesmo a nova sociologia económica?", Revista Crítica de Ciências Sociais, 73: 111-129.

Giner, Salvador (2010), El Futuro del Capitalismo, Barcelona, Ediciones Península.
Granovetter, Mark (1990), "The old and the new economic sociology: a history and an agenda", en Roger Friedland y A. F. Robertson (orgs.), Beyond the Marketplace: Rethinking Economy and Society, New York, Aldine de Gruyter.

Guespin-Michel, Janine y Jacq, Annick (coord.) (2006), Le Vivant, entre Societé et Marché: une démocratie à inventer, Paris, Éditions Syllepse e Espaces Marx.

Jorge, Maria Manuel Araújo (1995), Biologia, Informação e Conhecimento, Lisboa, Fundação Calouste Gulbenkian e Junta Nacional de Investigação Científica.

Keller, Evelyn Fox (2000), The Century of the Gene, Cambridge, MA, Harvard University Press.

Kline, J.; Rosenberg, N. (1986), "An overview of innovation", en R. Landau y N. Rosenberg (eds.), The Positive Sum Strategy: Harnessing Technology for Economic Growth, Washington DC, National Academy Press, 275-305.

Krimsky, Sheldon (2003), Science in the Private Interest. Has the Lure of Profits Corrupted Biomedical Research?, Oxford, Rowman \& Littlefield.

Lamo de Espinosa, Emilio (2010), La Sociedad del Conocimiento. Información, Ciencia, Sabiduría, Madrid, Real Academia de Ciencias Morales y Políticas.

Leite, Marcelo (2006), Promessas do Genoma, São Paulo, Editora UNESP.

Lewontin, Richard (1998), Biologia como Ideologia, Lisboa, Relógio D'Água.

Nowotny, Helga; Pestre, Dominique; Schmidt-Aßmann, Eberhard; Schulze-Fielitz, Helmuth y Trute, Hans-Heinrich (2005), The Public Nature of Science under Assault: Politics, Markets, science and the law, Springer.

Nowotny, Helga et al. (2001), Re-Thinking Science: knowledge and the public in age of uncertainty, London, Polity Press e Blackwell Publishers.
Oyama, Susan (2000), The Ontogeny of Information, Durham, NC, Duke University Press.

Pestre, Dominique (2003), Science, Argent et Politique. Un essai d'interpretation, Paris, INRA.

Polanyi, Karl (2000 [1944]), A Grande Transformação. As origens na nossa época, São Paulo, Elsevier e Editora Campus.

Polanyi, Karl (1994), "Nuestra obsoleta mentalidad de mercado", Cuadernos de Economía, Vol. XIV, No. 20, Universidad Nacional de Colombia, Bogotá, 249-266.

Schrödinger, Erwin (1989 [1944]), O que é a Vida? Espirito e matéria, Lisboa, Fragmentos.

Shannon, Claude y Weaver, Warren (1949), The Mathematical Theory of Communication, Urbana, Illinois, The University of Illinois Press.

Smelser, Neil y Swedeberg, Richard (orgs.) (1994), The Handbook of Economic Sociology, Princeton, New Jersey, Russel Sage Foundation.

Schumpeter, J. A. (1976), Capitalism, Socialism and Democracy, London, Routledge.

Schumpeter, J. A. (1996), Ensaios, Empresários, Inovação, Ciclos de Negócio e Evolução do Capitalismo, Oeiras, Celta Editores.

Ségalat, Laurent (2009), La Science à Bout de Soufflé?, Paris, Seuil.

Shinn, Terry y Ragouet, Pascal (2008), Controvérsias sobre a Ciência. Por uma sociologia transversalista da atividade Científica, São Paulo, Editora 34.

Sunder Rajan, K. (2006), Biocapital: the constitution of postgenomic life, Durham, Duke University Press.

Swedberg, Richard (2003), Principles of Economic Sociology, Princeton, Princeton University Press.

Thacker, E. (2005), The Global Genome: biotechnology, politics, and culture, Cambridge, MA, MIT Press. 
von Hippel, Eric (2006), Democratizing Innovation, Cambridge, MA, The MIT Press.

Weingart, P. (1997), "From 'Finalization' to 'Mode 2': old wine in new bottles?", Social Science Information, vol. 36, 4: 591-613.
Wiener, Norbert (1948), Cybernetics, Cambridge, MIT Press.

Ziman, J. (1996), "Postacademic science: constructing knowledge with networks and norms", Science Studies, vol. 9, 1: 67-80.
Ziman, J. (1994), Prometheus Bond. Science in a steady state, Cambridge, Cambridge University Press.

Zvick, Detlev et al. (2008), "Putting consumers to work: 'co-creation' and new marketing governmentality", Journal of Consumer Culture, 8. 